\title{
SUR LES ENSEMBLES INFINIS ET LINÉAIRES \\ DE POINTS
}

\author{
PAR
}

G. CANTOR

a HAL L $\mathbf{E}$ a. $\mathrm{S}$.

. I.

(Extrait des Annales mathématiques de Leipsic, vol. 15.)

Dans un mémoire publié dans le Journal de M. Borchardt, t. 84 j'ai démontré pour une classe très-étendue d'ensembles géométriques et arithmétiques, soit continus, soit discontinus, qu'on peut les faïre correspondre sans ambiguité à des points distribués d'une façon continue ou discontinue sur un segment de droite. Ces derniers ensembles acquièrent par là une importance particulière; nous les appellerons ensembles ou systêmes linéaires de points. Les points d'un tel systême sont distribués sur un segment de droite de longueur finie ou infinie ou bien de façon à occuper tout le segment ou bien de façon à n'occuper que des parties de ce segment, et il ne parait pas hors de propos de les étudier et de chercher les classer; c'est ce que nous nous proposons de faire ici. En partant de divers points de vue et des principes de classification, qui s'y rattachent, nous sommes amenés à partager les ensembles de points linéaires en certaines catégories. Pour commencer par un de ces points de vue, rappelons-nous la notion de l'ensemble dérivé d'un ensemble de points donné $P$, telle qu'elle a été donnée dans un travail sur les séries trigono- 
métriques (Annales math. t. 5); dans un ouvrage récemment paru de M. U. DINI (Fondamenti per la teorica d. funz. d. variabili reali, Pisa, 1878) nous voyons cette notion encore plus développée, puisqu'elle sert de point de départ à une série de généralisations remarquables de théorèmes analytiques connus. $\left({ }^{1}\right)$

D'ailleurs cette notion de l'ensemble dérivé d'un ensemble donné n'est pas restreinte aux ensembles linéaires, mais elle s'applique de la même. manière aux ensembles à deux, trois ou $n$ dimensions continus ou discontinus. Comme nous le montrerons plus tard, c'est sur cette notion que repose la conception la plus claire et en même temps la plus générale d'un ensemble continu.

Le dérivé $P^{(1)} d^{\prime} u n$ ensemble de points $P$ est en effet l'ensemble de tous les points qui jouissent de la propriété de coïncider avec un pointlimite de $P$, peu importe d'ailleurs que ce point-limite soit en même temps un point de $P$, ou non.

Comme alors le dérivé d'un ensemble $P$ est un nouveau ensemble déterminé $P^{(1)}$, on peut aussi chercher le dérivé de $P^{(1)}$, qui s'appellera le deuxième ensemble dérivé ou simplement le deuxième dérivé de $P$; et en continuant ainsi, on obtient le $\nu^{\text {eme }}$ dérivé de $P$, que l'on désigne par $P^{(\nu)}$.

Il peut se faire que la suite des dérivés $P^{(1)}, P^{(2)}, \ldots \ldots$ conduise à un dérivé $P^{(n)}$ composé de points qui ne se présentent qu'en nombre fini dans chaque étendue finie, en sorte que $P^{(n)}$ n'a pas de poirts-limites et par conséquent ne donne lieu à aucun dérivé; dans ce cas nous disons que le système de points $P$ est du premier genre et de la $n^{\text {éme }}$ espéce. Mais si la série des dérivés de $P$, la série $P^{(1)}, P^{(2)}, P^{(3)}, \ldots$ est infinie, nous disons que l'ensemble de points $P$ est du deuxième genre. De là on reconnaît sans peine que si $P$ est du premier genre et de la $n^{\text {ème }}$ espèce, $P^{(1)}, P^{(2)}, P^{(3)}, \ldots \ldots$ appartiennent aussi au premier genre et sont respectivement de la $\overline{n-1}^{\text {ème }}$, de la $\overline{n-2}^{\text {ème }}$, de la $\overline{n-3}^{\text {ème }}$ espèce; qu'ensuite si $P$ est dù deuxième genre, la même conséquence s'applique à tous les dérivés $P^{(1)}, P^{(2)}, \ldots$. Il est aussi à remarquer que tous les points de $P^{(2)}, P^{(3)}, \ldots$ sont toujours aussi des points du premier dérivé

( $\left.{ }^{1}\right)$ Voir aussi: Ascoli, Nouve richerche sulla serie di Fourier. Reale Academia dei Lincei $(1877-78)$. 
Sur les ensembles infinis et linéaires de points.

$P^{(1)}$, tandis qu'un point appartenant à $P^{(1)}$ n'est pas nécessairement un point de $P$.

On découvre ensuite des caractères importants d'un ensemble de points $P$, si l'on étudie la manière dont se comporte cet ensemble par rapport à un intervalle donné continu $(\alpha \ldots \beta)$, dont les points extrèmes sont considérés comme appartenant à l'intervalle mếme. Il peut se faire que quelques points ou même que tous les points de cet intervalle soient en même temps des points de $P$, ou bien qu'aucun point de $(\alpha \ldots \beta)$ n'appartienne à $P$; dans ce dernier cas nous disons que $P$ est tout entier en dehors de l'intervalle $(\alpha \ldots \beta)$.

Si $P$ est contenu dans l'intervalle $(\alpha \ldots \beta)$, en tout ou en partie, il peut se présenter un cas remarquable: c'est le cas où chaque intervalle $(\gamma \ldots \delta)$, si petit qu'il soit, compris dans $(\alpha \ldots \beta)$, contient des points de $P$. Dans ce cas nous dirons que $P$ est condensé dans tout l'intervalle $(\alpha \ldots \beta)$.

Comme exemples de systèmes de points ainsi condensés dans toute l'étendue de l'intervalle $(\alpha \ldots \beta)$, nous avons: $1^{\circ}$ tout ensemble de.points auquel appartiennent comme éléments tous les points de l'intervalle $(\alpha \ldots \beta) ; 2^{\circ}$ l'ensemble de points composé de tous les points dont les abscisses sont des nombres rationnels; $3^{\circ}$ le système de points composé de tous les points, qui ont pour abscisses les nombres rationnels de la forme $\pm \frac{2 n+1}{2^{m}}$, où $n$ et $m$ sont des nombres entiers positifs.

De cette explication de l'expression scondensé dans toute l'étendue d'un intervalle donnén, il résulte que, sị un système de points n'est pas condensé dans. tout un intervalle $(\alpha \ldots \beta)$, il doit nécessairement exister un intervalle $(r \ldots o)$ compris dans le premier et où ne se trouve aucun point de $P$. On peut montrer aussi que, si $P$ est condensé dans tout l'intervalle $(\alpha \ldots \beta)$ non seulement la même chose est vrai pour $P^{(1)}$, mais encore $P^{(1)}$ a pour points tous ceux de l'intervalle $(\alpha \ldots \beta)$. On pourrait prendre cette propriété de $P^{(1)}$ comme point de départ de l'explication de l'expression sêtre condensé dans toute l'étendue d'un intervalle», puisqu'on peut dire: un système de points $P$ est condensé dans toute l'étendue de l'intervalle $(\alpha \ldots \beta)$ quand son premier dérivé $P^{(1)}$ renferme comme éléments tous les points de $(\alpha \ldots \beta)$.

Si $P$ est condensé dans tout un intervalle" $(\alpha \ldots \beta)$, il l'est aussi 
dans toute l'étendue d'un autre intervalle quelconque $\left(\alpha^{\prime} \ldots \beta^{\prime}\right)$ contenu dans le premier.

Un système de points $P$ condensé dans toute l'étendue d'un intervalle $(\alpha \ldots \beta)$ est nécessairement du deuxièmé genre; car alors $P^{(1)}$, et par suite $P^{(2)}, P^{(3)} \ldots$ sont aussi condensés dans tout lintervalle $(\alpha \ldots \beta)$ et cette suite de dérivés de $P$ est illimitée, c. a. d. que $P$ appartient au second genre.

De là nous concluons qu'un système de points $P$ du premier genre n'est sùrement pas condensé dans tout un intervalle $(\alpha \ldots \beta)$, quel que soit d'ailleurs cet intervalle et que, par suite, on peut toujours trouver dans $(\alpha \ldots \beta)$ un intervalle $(\gamma \ldots \delta)$, qui ne renferme pas un seul point de $P$.

Quant à la question de savoir si réciproquement tout système de points du deuxièrne genre est de telle nature, qu'il y ait un intervalle dans toute l'étendue duquel il soit condensé, nous nous en occuperons plus tard. Nous arrivons maintenant à un second mode de classification des ensembles linéaires de points non moins important que le premier: il est fondé sur la considération de la puissance. Dans le mémoire cité plus haut (Journal de M. Borchardt t. 84) nous avons dit en général de deux ensembles $M$ et $N$ géométriques, arithmétiques ou appartenant à quelque autre catégorie bien définie, qu'ils ont même puissance, quand on peut les faire correspondre entre eux d'après quelque loi déterminée, de manière qu'à chaque élément de $M$ corresponde un élément de $N$ et, réciproquement, à chaque élément de $N$, un élément de $M$.

Suivant que deux ensembles sont de même puissance ou non, elles peuvent être placés dans une même classe ou dans des classes différẹntes. On peut appliquer ces règles générales spécialement aux ensembles linéaires de points que l'on partagera par conséquent en classes déterminées; les systèmes de points d'une classe sont tous de même puissance, au contraire les systèmes de points appartenant à différentes classes sont de puissance différente. Chaque système de points particulier peut ètre considéré comme représentant la classe à laquelle il appartient. Ici se présente en première ligne la classe des systèmes de points qui ont la même puissance que la suite naturelle des nombres: $1,2,3, \ldots \nu, \ldots$ et qu'on peut, par conséquent, représenter sous forme d'une série simplement infinie, dont le terme général dépend de $\nu$. 
A cette première classse appartiennent par exemple tous les systemes de points du premier genre; mais beaucoup de systèmes de points du deuxième genre font aussi partie de cette classe, par exemple: $1^{\circ}$ le système de points composé de tous les points d'un intervalle qui ont pour abscisses des nombres rationnels (cf. Journal d. M. Borchardt, t. 84, p. $250) ; 2^{\circ}$ le système de points composé de tous les points d'un intervalle qui ont pour abscisses des nombres algébriques (cf. Journal de M. BoRCHARDT, t. 77, p. 258).

Après cela, se présente à nous une seconde classe de systèmes linéaires de points; cette classe est représentée par le système des points appartenant à un intervalle continu, par exemple par le système de tous les points dont les abscisses sont $\geqq 0$ et $\leqq 1$. A cette classe appartiennent par exemple:

$1^{\circ}$ Tout intervalle continu $(\alpha \ldots \beta)$.

$2^{\circ}$ Tout système de points composé de plusieurs intervalles séparés continus $(\alpha \ldots \beta),\left(\alpha^{\prime} \ldots \beta\right),\left(\alpha^{\prime \prime} \ldots \beta^{\prime \prime}\right), \ldots$, en nombre fini ou infini.

$3^{\circ}$ Tout système de points obtenu en supprimant dans un intervalle continu un ensemble fini ou infini de points $u_{1}, u_{2}, \ldots u_{\nu}, \ldots$ de la première classe (cf. Journal de M. BoRChaRdT, t. 84, p. 254).

- Nous n'examinerons pas ici, si ces deux classes sont les seules que forment les ensembles infinis et linéaires de points; mais nous voulons démontrer maintenant que ces deux classes sont distinctes en réalité; pour cela il faut d'abord montrer qu'on ne peut pas faire correspondre entre eux point pour point deux représentants quelconques de ces deux classes.

Comme représentant de la deuxième classe choisissons ici l'intervalle continu $(0 \ldots 1)$; si cet ensemble appartenait en mème temps à la première classe, il devrait exister une série simplement infinie $u_{1}, u_{2}, \ldots u_{\nu}, \ldots$ composée de tous les nombres réels $\geqq 0$ et $\leqq 1$, en sorte que tout nombre situé dans cet intervalle se présenterait dans cette série à une place déterminée. Mais cette hypothèse est en contradiction avec un théorème trèsgénéral que nous avons démontré rigoureusement dans le journal de M. BoRCHARDT, t. 77, p. 260, à savoir:

„Etant donnée une série simplement infinie 
de nombres réels inégaux progressant d'après une loi quelconque, on peut indiquer dans chaque intervalle proposé $(\alpha \ldots \beta)$ un nombre $v$ (et par conséquent on en peut indiquer une infinité) qui ne soit pas compris parmi les termes de cette série.»

En égard au grand intérét qui s'attache à ce théorème, non seulement dans la présente théorie, mais encore dans beaucoup d'autres questions d'arithmétique ou d'analyse, il ne parait pas inutile de développer ici, en la modifiant et la simplifiant, la démonstration que noưs avons donnée alors.

D'après cela, étant donnée une série

$$
u_{1}, u_{2}, \ldots u_{\nu}, \ldots
$$

que nous désignerons par le symbole $\left(u_{\nu}\right)$ et un intervalle quelconque $(\alpha \ldots \beta)$ où $\alpha<\beta$; il s'agit de démontrer que, dans cet intervalle on peut trouver un nombre réel $v$, qui ne se présente pas dans $\left(\boldsymbol{u}_{v}\right)$.

I. Nous remarquons d'abord que si notre ensemble $\left(u_{\nu}\right)$ n'est pas condensé dans tout l'intervalle $(\alpha \ldots \beta)$, il faut que dans cet intervalle $(\alpha \ldots \beta)$ il $\mathrm{y}$ en ait un autre $\left(\gamma \ldots \delta^{\prime}\right)$ dont tous les nombres n'appartiennent pas à $\left(u_{v}\right)$; on peut donc choisir pour $v$ un nombre quelconque de l'intervalle $(\gamma \ldots \delta)$. Ce cas ne présente donc aucune difficulté et nous pouvons passer à l'autre cas plus compliqué.

II. Supposons l'ensemble $\left(u_{v}\right)$ condensé dans tout l'intervalle $(\alpha \ldots \beta)$. Dans ce cas tout intervalle $(\gamma \ldots \delta)$, si petit qu'il soit, compris dans $(\alpha \ldots \beta)$ contient des nombres de notre série $\left(u_{\nu}\right)$. Pour montrer que néanmoins il y a dans l'intervalle $(\alpha \ldots \beta)$ des nombres $v$ qui ne se trouvent pas dans $\left(u_{\nu}\right)$, faisons les remarques suivantes. Comme dans notre série:

$$
u_{1}, u_{2}, \ldots u_{v}, \ldots
$$

il y a certainement des nombres qui se rencontrent dans l'intervalle $(\alpha \ldots \beta)$, il faut qu'un de ces nombres ait le plus petit indice, qu'il soit $u_{x_{1}}$, et un autre l'indice immédiatement supérieur: $u_{x_{2}}$.

Désignons par $\alpha^{\prime}$ le plus petit des deux nombres $u_{x_{1}}, u_{x_{2}}$, et le plus grand par $\beta$.

(Ils ne peuvent être égaux entre eux, parce que nous avons supposé notre série composée de nombres inégaux.)

On a alors d'aprés la définition:

$$
\alpha<\alpha^{\prime}<\beta^{\prime}<\beta,
$$


Sur les ensembles -infinis et linéaires de points.

puis: $x_{1}<x_{2}$; et il faut remarquer en outre que tous les nombres $u_{\mu}$ de notre série pour lesquels $\mu \leqq x_{2}$ ne sont pas situés à l'intérieur. de l'intervalle $\left(\alpha^{\prime} \ldots \beta^{\prime}\right)$ comme il ressort immédiatement de la détermination des nombres $u_{x_{1}}, u_{x_{2}}$. De même désignons par $u_{x_{3}}, u_{x_{4}}$ les deux nombres de la série $\left(u_{\nu}\right)$ affectés des plus petits indices, que l'on rencontre situés dans les limites de l'intervalle $\left(\alpha^{\prime} \ldots \beta^{\prime}\right)$; soit $\alpha^{\prime \prime}$ le plus petit de ces nombres et $\beta^{\prime \prime}$ le plus grand.

On a alors:

$$
\begin{gathered}
\alpha^{\prime}<\alpha^{\prime \prime}<\beta^{\prime \prime}<\beta^{\prime}, \\
x_{2}<x_{3}<x_{4},
\end{gathered}
$$

et on reconnait que tous les nombres $u_{\mu}$ de notre série pour lesquels $\mu \leqq x_{4}$ ne sont pas compris dans l'intérieur de l'intervalle $\left(\alpha^{\prime \prime} \ldots \beta^{\prime \prime}\right)$.

Quand on est arrivé, en suivant toujours la même loi, $\grave{a}$ un intervalle $\left(\alpha^{(\nu-1)} \ldots \beta^{(\nu-1)}\right)$, l'intervalle suivant se tire de ce dernier, en considérant les deux premiers nombres de notre série $\left(u_{\nu}\right)$ (c. à d. ceux qui ont les plus petits indices) qui se rencontrent dans l'intervalle $\left(\alpha^{(\nu-1)} \ldots \beta^{(\nu-1)}\right)$; soient $u_{x_{2 \nu-1}}, u_{x_{2 \nu}}$ ces deux nombres; on désignera le plus petit d'entre eux par $\alpha^{(\nu)}$, le plus grand par $\beta^{(\nu)}$.

L'intervalle $\left(\alpha^{(\nu)} \ldots \beta^{(\nu)}\right)$ est alors compris dans tous les intervalles précédents et il a avec notre série $\left(u_{v}\right)$ ce rapport particulier que tous les nombres $u_{\mu}$ pour lesquels $\mu ₹ \chi_{2 \nu}$, ne sont certainement pas compris dans cet intervalle. Comme évidemment:

$$
x_{1}<x_{2}<x_{3}<\ldots, x_{2 \nu-2}<x_{2 \nu-1}<x_{2 \nu}, \ldots
$$

et que ces nombres, en tant qu'indices, sont des nombres entiers, on a:

$$
x_{2 \nu} \equiv 2 \nu
$$

et par suite:

$$
\nu<x_{2 \nu}
$$

nous pouvons donc assurer, et cela nous suffit pour ce qui doit suivre, que: $\nu$ étant un nombre entier pris arbitrairement, la grandeur $u_{\nu}$ est en dehors de l'intervalle $\left(\alpha^{(\nu)} \ldots \beta^{(\nu)}\right)$.

Comme les nombres $\alpha^{\prime}, \alpha^{\prime \prime}, \alpha^{\prime \prime \prime}, \ldots \alpha^{(\nu)}, \ldots$ augmentent constamment de grandeur et sont néanmoins renfermés dạns l'intervalle $(\alpha \ldots \beta)$, ils 
ont, d'après un théorème fondamental bien connu de la théorie des grandeurs, une limite que nous désignons par $A$, en sorte que:

$$
A=\lim \alpha^{(\nu)} \quad \text { pour } \nu=\infty .
$$

La même chose est vraie pour les nombres $\beta^{\prime}, \beta^{\prime \prime}, \beta^{\prime \prime \prime}, \ldots \beta^{(\nu)}, \ldots$ qui décroissent constamment tous en restant compris dans l'intervalle $(\alpha \ldots \beta)$; nous désignerons leur limite par $B$, en sorte que:

$$
B=\lim \beta^{(v)} \quad \text { pour } \nu=\infty .
$$

On a évidemment:

$$
\alpha^{(\nu)}<A \leqq B<\beta^{(\nu)} .
$$

Mais il est facile de voir que le cas $A<B$ ne peut se présenter ici; autrement comme tout nombre $u_{\nu}$ de notre série serait en dehors de l'intervalle $(A \ldots B)$, puisque $u_{\nu}$ est en dehors de l'intervalle $\left(\alpha^{(\nu)} \ldots \beta^{(\nu)}\right)$, notre série $\left(u_{\nu}\right)$ ne serait pas condensé dans tout l'intervalle $(\alpha \ldots \beta)$, contrairement à la supposition que nous avons faite.

Il ne reste donc que le cas $A=B$ et il est évident maintenant que le nombre: $v=A=B$ ne se présente pas dans notre série $\left(u_{\nu}\right)$.

Car si ce nombre était membre de notre série, soit le $\nu^{\text {dme }}$, on aurait: $v=u_{\nu}$.

Mais cette dernière équation n'est possible pour aucune valeur de $\nu$, parce que $v$ est compris dans l'intervalle $\left(\alpha^{(\nu)} \ldots \beta^{(\nu)}\right)$, tandis que $u_{\nu}$ est en dehors de ce même intervalle.

Halle a. S. Janvier 1879. 


\title{
SUR LES ENSEMBLES INFINIS ET LINÉAIRES
}

\section{DE POINTS}

\author{
PAR \\ G. CANTOR

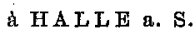 \\ II. \\ (Extrait des Annales mathém. de Leipsic, t. 17.)
}

Pour faciliter, en l'abrégeant, l'exposition 'qui va suivre, qu'on me permette d'indiquer tout d'abord un système de notations.

Nous exprimerons par la formule $P \equiv Q$ l'identité de deux systèmes de points $P$ et $Q$. Si les deux systèmes $P$ et $Q$ n'ont aucun élément commun nous dirons qu'ils sont sans connexion. Si un système $P$ est composé de la réunion de plusieurs systèmes $P_{1}, P_{2}, P_{3}, \ldots$, en nombre fini ou infini, n'ayant deux à deux aucune connexion, nous écrirons:

$$
P \equiv\left(P_{1}, P_{2}, P_{3}, \ldots\right) \text {. }
$$

Si tous les points d'un système $P$ appartiennent à un autre système $Q$, nous dirons que $P$ est contenu dans $Q$ ou encore que $P$ est un diviseur de $Q, Q$ un multiple de $P$. Soient $P_{1}, P_{2}, P_{3}, \ldots$ des systèmes de points quelconques en nọmbre fini ou infini; ces systèmes ont un plus petit commun multiple que nous désignons par $M\left(P_{1}, P_{2}, P_{3}, \ldots\right)$; ce plus petit commun multiple est le système composé de tous les points différents de $P_{1}, P_{2}, P_{3}, \ldots$ et n'ayant pas d'ailleurs d'autres points comme éléments; ces systèmes ont de même un plus grand commun diviseur que nous désignons par $\mathfrak{D}\left(P_{1}, P_{2}, P_{3}, \ldots\right)$ et qui est le système des points communs à tous les $P_{1}, P_{2}, P_{3}, \ldots$ Par exemple, $P^{(1)}, P^{(2)}, P^{(3)}, \ldots$ 
étant les dérivés successifs d'un système de points $P$ (v. Art. I), nous pouvons dire que $P^{(2)}$ est diviseur de $P^{(1)}, P^{(3)}$ diviseur de $P^{(2)}$ aussi bien que de $P^{(1)}$, et en général $P^{(\nu)}$ diviseur de $P^{(\nu-1)}, P^{(\nu-2)}, \ldots P^{(1)}$; au contraire $P^{(1)}$ en général n'est pas diviseur de $P$; mais si $P$ lui-même est le premier dérivé d'un système $Q, P^{(1)}$ sera diviseur de $P$.

Il est de plus utile d'avoir un signe qui exprime l'absence de points; nous choisissons pour cela la lettre 0 ; ainsi $P \equiv 0$ signifie que le système $P$ ne contient pas un seul point, et qu'ainsi, rigoureusement parlant, ce n'est pas un vrai système. Pour en donner ici un exemple, un système de points du premier genre et de la $n^{\text {ème }}$ espèce est caractérisé par $P^{(n+1)} \equiv 0$, au contraire $P^{(n)}$ est différent de 0 .

Deux systèmes sont en connexion par leur plus grand commun diviseur, et si ce dernier $\equiv 0$, ils sont sans connexion.

Si deux systemes de points $P$ et $Q$ ont la même puissance et appartiennent par conséquent à une même classe (art. I), nous les appelons équivalents et nous exprimons cette relation par la formule:

$$
P \sim Q \text {. }
$$

Si on a: $P \sim Q ; Q \sim R$, on aura toujours aussi: $P \sim R$.

Soient ensuite $P_{1}, P_{2}, P_{3}, \ldots$ une série de systèmes, qui pris deux à deux n'ont aucune connexion entre eux, $Q_{1}, Q_{2}, Q_{3}, \ldots$ une autre série dans les mèmes conditions; soit aussi: $P_{1} \sim Q_{1} ; P_{2} \sim Q_{2} ; P_{3} \sim Q_{3} ; \ldots$, on aura:

$$
\left(P_{1}, P_{2}, P_{8}, \ldots\right) \sim\left(Q_{1}, Q_{2}, Q_{3}, \ldots\right) \text {. }
$$

Les systèmes de points du premier genre, comme nous venons de le voir, peuvent être caracterisés d'une manière complète par la notion du système dérivé, telle qu'elle a été développée jusqu'ici; pour ceux du second genre cette notion ne suffit plus, et il faut en donner ici une extension qui se présente comme d'elle-même quand on approfondit la question.

Remarquons que dans la série des dérivés $P^{(1)}, P^{(2)}, P^{(3)}, \ldots$ d'un système $P$, chaque terme est diviseur des précédents, et que par suite chaque nouveau dérivé $P^{(\nu)}$ se tire du précédent $P^{(\nu-1)}$ par l'élimination de certains points, sans qu'il s'en rencontre de nouveaux. 
Si $P$ est du deuxième genre, $P^{(1)}$ se composera de deux systèmes de points $Q$ et $R$ essentiellement distincts, en sorte que: $P^{(1)} \equiv(Q, R)$; l'un; $Q$, se compose des points de $P^{(1)}$ qui disparaissent dans la série $P^{(1)}, P^{(2)}$, $P^{(3)}, \ldots$ quand on a suivi cette marche assez longtemps; l'autre $R$ comprend les points qui sont conservés dans tous les termes de la série $P^{(1)}$, $P^{(2)}, P^{(3)}, \ldots ; R$ est donc défini pạr la formule:

$$
R \equiv \mathfrak{D}\left(P^{(1)}, P^{(2)}, P^{(3)}, \ldots\right) .
$$

Mais nous avons aussi évidemment:

et en général:

$$
R \equiv \mathfrak{D}\left(P^{(2)}, P^{(3)}, P^{(4)}, \ldots\right)
$$

$$
R \equiv \mathfrak{D}\left(P^{\left(n_{1}\right)}, P^{\left(n_{2}\right)}, P^{\left(n_{3}\right)}, \ldots\right),
$$

où $n_{1}, n_{2}, n_{3}$ est une suite quelconque de nombres entiers positifs croissant à l'infini.

Désignons maintenant par le signe $P^{(\omega)}$ ce système de points $R$ obtenu ainsi à l'aide du système $P$, et appelons-le le système dérivé de $P$ d'ordre $\omega$.

Désignons par $P^{(\omega+1)}$ le premier dérivé de $P^{(\omega)}$, par $P^{(\omega+n)}$ le $n^{\text {ème }}$ dérivé de $P^{(\omega)} ; P^{(\omega)}$ aura aussi un système dérivé d'ordre $\omega$ généralement distincte de 0 , et que nous appellerons $P^{(2 \omega)}$. En continuant ces opérations, on arrive $\grave{a}$ des dérivés que nous désignerons conséquemment par: $P^{\left(n_{0} \omega+n_{1}\right)}$, où $n_{0}, n_{1}$ sont des nombres entiers positifs. Mais nous pouvons aller plus loin et former le système:

$$
\mathfrak{D}\left(P^{(\omega)}, P^{(2 \omega)}, P^{(3 \omega)}, \ldots\right)
$$

qui ser $x$ désigné par le symbole $P^{\left(w^{2}\right)}$.

En répétant maintenant la même opération et en la combinant avec les précédentes, on arrive à une notion plus générale, celle du système dérivé:

$$
P^{\left(n_{0} \omega^{2}+n_{2} \omega+n_{2}\right)},
$$

et en poursuivant cette marche on arrive à:

$$
\left.\boldsymbol{P}^{\left(n _ { 0 } \left(\omega^{\nu}+n_{1} \omega^{\nu^{\nu}}-1\right.\right.}+\ldots+n_{\nu}\right),
$$

où $n_{0}, n_{1}, \ldots n_{y}$ sont des nombres entiers positifs. En continuant cette généralisation on est amené à considérer $\nu$ comme variable et à envisager le système:

$$
P^{\left(\omega^{\omega}\right)} \equiv \mathfrak{D}\left(P^{(\omega)}, P^{\left(\omega^{2}\right)}, P^{\left(\omega^{3}\right)}, \ldots\right)
$$


On obtient successivement, en continuant de même, la notion de systèmes dérivés désignés par:

$$
P^{\left(n \omega^{\omega}\right)}, P^{\left(\omega^{\omega \omega+1}\right)}, P^{\left(\omega^{\omega+n}\right)}, P^{\left(\omega^{n \omega}\right)}, P^{\left(\omega^{\omega^{n}}\right)}, P^{\left(\omega^{\left(\omega^{\omega}\right)}\right)} \text { etc. }
$$

Nous avons ainsi une suite infinie de systèmes, qui se déduisent les uns des autres suivant une loi nécessaire et indépendamment de toute conception arbitraire.

On a, pour les systèmes de points du premier genre, comme il résulte de leur définition même:

$$
P^{(w)} \equiv 0
$$

il est à remarquer qu'on peut démontrer aussi la réciproque: tout système de points pour lequel cette équation a lieu, est du premier genre; les systèmes du premier genre, sont donc complètement caractérisés par cette équation.

Il est facile d'imaginer l'exemple d'un système de points du deuxième genre, pour lequel $P^{(\omega)}$ est composé d'un point donné $p$. A cet effet, considérons des intervalles qui se suivent, se limitent mutuellement, et convergent en même temps vers le point $p$ en devenant infiniment petits; prenons dans chacun de ces intervalles un système de points du premier genre, dont l'ord́re croisse au delà de toute limite, quand l'intervalle correspondant se rapproche de $p$. La reunion de tous ces systèmes fournit l'exemple en question. Cet exemple résout en mêrne temps la question, posée dans l'art. I, de savoir si, à un système de points du deuxième genre, doit toujours appartenir un intervalle dans. toute l'étendue duquel il soit condensé; or nous voyons, par l'exemple indiqué que cela n'a pas lieu nécessairement.

On construit avec la même facilité des systèmes de points du deuxième genre, pour lesquels $P^{(\omega+n)}$ ou $P^{(2 \omega)}$ ou plus généralement:

$$
\left.P^{\left(n_{0} \omega^{y}+n_{1} \omega^{\nu}-1\right.}+\ldots+n_{\nu}\right)
$$

ce composent d'un point $p$ déterminé d'avance.

Pour tous les systèmes analogues, il n'existe aucun intervalle dans toute l'étendue duquel ils soient condensés; de plus tous ces systèmes appartiennent à la première classe; à ce double point de vue ils ressemblent aux systèmes de points du premier genre.

Halle, mai 1880. 


\section{SUR LES ENSEMBLES INFINIS ET LINEAIRES \\ DE POINTS}

PAR

G. CANTOR

b HALLE a. S.

\section{III.}

(Extrait d'un mém. d. Annales math. cle Leipsic, t. XX, p. 113.)

Dans les deux articles précédents nous nous en sommes tenus rigoureusement au sujet indiqué par notre titre, et nous nous-sommes occupés exclusivement de systèmes de points linéaires, c. à d. d'ensembles de points, donnés d'après une certaine loi et appartenant à une ligne droite continue indéfinie. C'était à dessein que je m'étais borné à ce cas; en effet, d'après les résultats indiqués dans mon travail: Contribution à la théorie des ensembles, (Journal de Borchardt, t. 84, p. 242), l'on peut faire correspondre, sans ambiguité, élément par élément, des ensembles à deux, trois, ... $n$ dimensions à des systèmes linéaires de points; et l'on peut admettre a priori que la plupart des propriétés et des relations trouvées pour les ensembles linéaires de points, peuvent se démontrer aussi, avec des modifications faciles à deviner, pour les systèmes de points contenus dans des surfaces ou des espaces continus ou dans des ensembles continus de $n$ dimensions. Mais je voudrais maintenant exposer cette généralisation d'une manière plus précise; car elle n'est pas seulement intéressante en ellemème et au point de vue des applications qu'on en peut faire dans la 
théorie des fonctions, mais elle fournit encore de nouveaux points de vue pour l'étude des ensembles linéaires de points.

Pour commencer par un de ces points de vue, on peut étendre immédiatement aux systèmes de points que l'on rencontre dans des ensembles continus de $n$ dimensions, les notions précédemment données sur les dérivés des divers ordres déterminés nous seulement par des nombres entiers finis, mais caractérisés dans certains cas par des symboles infinitaires dont la signification a été rigoureusement fixée. La notion de l'ensemble dérivé s'appuie encore ici sur celle de point-limite d'un ensemble de points donné $P$; et ce point-limite est défini par cette condition que, dans un espace aussi petit que l'on veut entourant ce point, il y a des points du système $P$ autres que ce point lui-même; d'après cette définition le point-limite peut indifféremment appartenir ou ne pas appartenir au système $P$. M. Weierstrass a le premier enoncé d'une manière générale, et aṕliqué à la théorie des fonctions, le théorème suivant: tout système de points composé d'un nombre infini de points et situé dans une portion finie et continue d'un ensemble à $n$ dimensions a au moins un point-limite.

L'ensemble de tous les points-limites d'un système $P$ forme un nouveau système de points $P^{\prime}$, généralement distinct de $P$, et que j'appelle premier dérivé de $P$. On tire de là les notions des dérivés d'ordre plus élevé en reproduisant cette mểme opération un nombre fini ou même infini de fois. A l'égard de ces dérivés successifs se présente toujours ce fait facile à expliquer que tout dérivé, excepté le premier, est contenu dans les ensembles précédents, y compris le premier dérivé $P$; tandis que le système donné $\boldsymbol{P}$ contient en général des points qui n'appartiennent pas à ses dérivés. On peut de même appliquer immédiatement aux systèmes à plusieurs dimensions la notion de la condensation dans un intervalle, que nous n'avons considérée d'abord que par rapport aux systèmes linéaires de points. Etant donné un système de points $P$ situé dans un ensemble continu $G_{n}$ à $n$ dimensions, noụ dirons que ce système est condensé dans toute l'étendue d'un ensemble continu partiel a contenu dans $G_{n}$, si tout ensemble $a^{\prime}$ contenu dans $a$ et ayant le même nombre de dimensions que a renferme des points du système $P$.

Le premier dérivé $P^{\prime}$ (et de même tous les suivants) d'un système de points $P$ condensé dans toute l'étendue d'un ensemble continu $a$ renferme l'ensemble continu $a$ lui-même avec tous les points de la limite du 
dernier; et réciproquement on peut aussi prendre cette propriété du système de points $P$ comme point de départ pour arriver à la définition de la condensation de ce système dans toute l'étendue de l'ensemble $a$.

De même la notion de puissance, qui renferme en elle-même, comme cas particulier, la notion du nombre entier, ce fondement de la théorie des grandeurs, et que l'on pourrait considérer dans les ensembles comme le moment le plus général, cette notion, dis-je, est loin d'être restreinte aux systèmes de points linéaires; on peut bien plutôt la considérer comme un attribut de tout ensemble bien défini, quelle que soit d'ailleurs, la constitution de ses éléments.

Je dis qu'un ensemble d'éléments appartenant à une sphère abstraite quelconque, est bien défini quand, par suite du principe logique du troisième exclu, on peut le considérer déterminé de telle façon que $1^{\circ}$ un objet quelconque appartenant à. cette sphère abstraite étant choisi, l'on puisse regarder comme intrinsèquement déterminé s'il appartient ou non au système en question et que $2^{\circ}$ deux objets appartenant à l'ensemble étant donnés l'on puisse regarder comme intrinsèquement déterminé s'ils sont égaux ou non, malgré les différences qui peuvent se présenter dans la manière dont ils sont donnés.

En fait, on ne pourra pas généralement effectuer d'une manière sùre et précise les déterminations en question avec les méthodes ou les moyens dont on dispose; mais là n'est pas la question; il ne s'agit que de la détermination intrinsèque dont il faut tirer une détermination actuelle (extrinsèque) en perfectionnant les moyens auxiliaires, dans des cas concrets où cela sera nécessaire.

Pour éclaircir ceci, je rappelle la définition du système de tous les nombres algébriques; on peut, sans aucun doute, le concevoir ètre déterminé intrinsèquement si un nombre $\eta$ choisi à volonté appartient ou non aux nombres algébriques; néanmoins le problème qui consiste à trouver cette dẹtermination par rapport à un nombre donné $\eta$, est souvent, comme on le sait, un des plus difficiles; et c'est encore par exemple une question toujours indécise, et du plus haut intérêt, de savoir si le nombre $\pi$, qui exprime le rapport de la circonférence au diamètre, est un nombre algébrique, ou, comme c'est beaucoup plus vraisemblable, un nombre transcendant. Le même problème a été résolu il $\mathrm{y}$ a huit ans par $\mathrm{M}$. Crr. Hermite, pour le nombre fondamental $e$ du système naturel de loga- 
rithmes, dans le travail remarquable: mSur la fonction exponentiellen, (Paris 1874); il y démontre que le nombre $e$ n'est racine d'aucune équation algébrique à coefficients rationnels entiers.

$\mathrm{Si}$ l'on a un ensemble géométrique, dont les éléments peuvent être non seulement des points, mais des lignes, des surfaces ou des solides, et si cet ensemble est bien défini, la question de sa puissance se présente encore immédiatement, et cette puissance sera ou égale à une des puissances que l'on rencontre dans des ensembles de points ou plus grande que toutes les puissances de ce genre.

Pour ce qui concerne les systèmes de points compris dans des ensembles continus de $n$ dimensions, j'ai démontré rigoureusement (Journal de Borchandt, t. 84, p. 242) que leurs puissances sont les mêmes que cell's des ensembles linéaires de points; ce fait peut être regardé-comme une simple conséquence du. théorème démontré dans ce Journal, et d'après lequel on peut faire correspondre élément par élément un ensemble continu à $n$ dimensions à un ensemble continu à une dimension et par conséquent à un continuum linéaire droit; la question des diverses puissances dans les systèmes de points peut donc, sans rien perdre de sa généralité, se poser seulement pour les systemes de points linéaires, comme je l'ai fait remarquer à la fin du travail cité tout à l'heure.

J'ai emprunté le mot: puissance, à J. STteinen qui l'a employé dans un sens tout à fait spécial, mais cependant toujours analogue, pour exprimer que deux figures, si on les fait correspondre entre elles par projection, sont dans un rapport tel qu'à chaque élément de l'une répond un élément de l'autre, et un seulement; dans la notion absolue de puissance, que l'on rencontre ici, on maintient, il est vrai, la relation réciproque à sens unique, mais on ne fait aucune restriction pour la loi de la correspondance, particulièrement en ce qui regarde la continuité et la discontinuité, en sorte qu'on attribue à deux systèmes la même puissance quand on peut d'après une loi quelconque, établir entre eux une correspondance réciproque à sens unique, et on he peut leur attribuer la même puissance qu'à cette condition; quand les deux systèmes sont bien definis, on peut regarder comme intrinsèquement déterminée la question de savoir s'ils ont même puissance ou non; mais la solution actuelle de cette question dans les cas concrets est souvent un des problèmes les plus difficiles. Ce n'est qu'après bien des essais infructueux que j'ai pu réussir, il y a huit ans, 
à l'aide d'un théorème démontré dans le Journal de Bonchando, t. 77, p. 260, et dans l'article I du présent travail, à prouver que le continuium linéaire n'a pas la même puissance que la série naturelle des nombres.

La théorie des ensembles ainsi conçue, (en ne considérant que ce qui est mathématique et en laissant de côté provisoirement les autres sphères abstraites), comprend l'arithmétique, la théorie des fonctions et la géométrie; ces parties de la science sont ainsi ramenées grâce à la notion de puissance à une unité commune. Le continu et le discontinu sont ainsi considérés au mème point de vue et se trouvent ramenées à une commune mesure.

La plus petite puissance que l'on puisse rencontrer genéralement dans des systèmes infinis, c. à d. composés d'un nombre infini d'éléments, est la puissance de la série des nombres entiers positifs rationnels; j’ai nommé les ensembles de cette classe systèmes qu'on peut compter à l'infini, ou simplement systèmes dénombrables; ce qui les caractérise, c'est qu'on peut les représenter (de bien des manières) sous la forme d'une série régulière simplement infinie:

$$
E_{1}, E_{2}, \ldots E_{\nu}, \ldots \ldots
$$

en sorte que chaque élément du système occupe une place déterminée de la série et que la série ne renferme pas d'autres membres que les éléments du système donné.

Chaque partie infinie d'un système dénombrable forme un nouveau système qu'on peut dénombrer à l'infini.

Etant donné un système fini ou infini mais dénombrable de systèmes $(E),\left(E^{\prime}\right),\left(E^{\prime \prime}\right), \ldots$. , dont chacun est respectivement dénombrable, le système produit par la réunion de tous les éléments de $(E),\left(E^{\prime}\right),\left(E^{\prime \prime}\right), \ldots \ldots$ jouira de la même propriété.

Ces deux propositions simples et faciles à prouver servent de base a l'étude des systèmes dénombrables. Aussi, l'on reconnait, comme je l'ai déjà fait remarquer souvent, que tous les systèmes donnés sous la forme d'une série $n$-tiplement infinie dont le terme général est $E_{\nu_{1}}, \nu_{\nu_{2}}, \ldots \ldots \nu_{n}$ (où $\nu_{1}, \nu_{2}, \ldots \nu_{n}$ peuvent prendre, indépendamment l'un de l'autre, toutes les valeurs numériques positives entières) sont des systèmes, susceptibles d'ètre dénombrés, c. à d. qu'on peut les représenter sous la forme 
de séries simplement infinies; mais à cette classe appartiennent aussi des systèmes, dont le terme général a la forme:

$$
E_{\nu_{1}, \nu_{2}}, \ldots \ldots \nu_{\mu},
$$

où $\mu$ peut aussi prendre toutes les valeurs numériques positives entières; l'ensemble de tous les nombres algébriques est un exemple particulièrement remarquable de cette dernière espèce d'ensembles (V. J. de BоrchardT, t. 77 , p. 258). L'arithmétique et l'algèbre présentent une quantité innombrable d'exemples de cette propriété; toutefois la géométrie n'en offre pas en moindre quantité. Le théorème suivant qui trouve plus d'une application élégante dans la théorie des nombres et dans celle des fonctions, pourra en fournir la preuve.

Soit, dans un espace continu $G_{n}$, de $n$ dimensions, ètendu à l'infini de tous côtés, un nombre infini d'ensembles partiels $(a)$, continus, $\left({ }^{1}\right)$ de $n$ dimensions, séparés l'un de l'autre et ne se touchant tout au plus qu'à leurs limites; je dis que le système $(a)$ d'ensembles partiels de cette espèce peut toujours être dénombré.

Il faut remarquer qu'ici on ne fait aucune supposition sur le partage et sur la grandeur de l'espace total des ensembles $a$; leur éténdue peut ètre aussi petite qu'on voudra, et ils pourront se rapprocher indéfiniment de tout point de $G_{n}$ qui ne leur appartient pas; le théorème est sans aucune exception, pourvu seulement que chaque ensemble partiel $a$ (tous les $a$ ayant $n$ dimensions d'après l'hypothèse) occupe un volume total déterminé (aussi petit que l'on voudra) et'que les divers' $a$ ne se rencontrent tout au plus qu'à leurs limites.

On peut démontrer ce théorème de la manière suivante: Je suppose qu'au moyen de rayons vecteurs réciproques on transforme l'espace infini, à $n$ dimensions $G_{n}$ en une figure $H_{n}$ à $n$ dimensions comprise à l'interieur d'un espace infini $G_{n+1}$ de $n+1$ dimensions, où $H_{n}$ est déterminé de telle sorte que ses points sont tous à une distance 1 d'un point fixe de l'espace $G_{n+1}$. (Pour le cas $n=1$ ce sera un cercle de rayon 1; pour le cas $n=2$, une sphère de rayon 1). A chaque ensemble partiel $a$ de $G_{n}$ à $n$ dimensions correspond un ensemble partiel $b$ de $H_{n}$ à $n$

(') Pour chaque figure continue on considère comme en faisant partie les points qui lui servent de limite. 
dimensions, et d'étendue déterminée; si maintenant on peut démontrer pour le système $(b)$ la propriété de pouvoir être dénombré, on en déduira, $\grave{a}$ cause de la correspondance réciproque à sens unique, la même propriété pour le système $(a)$.

Le système $(b)$ est susceptible d'ètre dénombré parce que le nombre des ensembles $b$, qui d'après leur étendue sont plus grands qu'un nombre $r$ donné à volonté, est nécessairement fini; car leur somme est plus pẹtite que le nombre

$$
\frac{2 \pi^{\frac{n+1}{2}}}{\Gamma\left(\frac{n+1}{2}\right)}
$$

c. ̀̀ d. plus petite que l'étendue de la figure $H_{n}$, dans laquelle les $b$ sont tous compris; il suit de là que l'on peut ordonner les ensembles $b$, d'après la grandeur de leur étendue, en une série simplement infinie, en sorte que les plus petits suivent les plus grands et finissent par devenir infiniment petits:

Le cas $n=1$ donne lieu au théorème suivant, qui est essentiel pour le développement de la théorie des ensembles linéaires de points: Tout ensemble d'intervalles $(\alpha \ldots \ldots \beta)$ distincts, ne se rencontrant tout au plus qu’à leurs points extrêmes, et situés sur une ligne droite indéfinie, est nécessairement un ensemble susceptible d'ètre dénombré; la même chose est donc vraie aussi pour le système des points extrêmes $\alpha$ et $\beta$, mais ne l'est pas toujours pour le dérivé du dernier ensemble de points.

Dans le cas $n=2$, ce théorème montre que l'on peut dénombrer tout ensemble de surfaces partielles distinctes, ne se rencontrant tout au plus qu’à leurs limites, et situées dans un plan indéfini; ce cas paraît avoir de l'importance dans la théorie des fonctions de variables complexes. Je remarque en même temps qu'il n'est pas difficile d'étendre ce théorème aux ensembles de surfaces partielles distinctes situées sur une surface qui recouvre le plan un nombre fini ou infini de fois.

Quant aux ensembles de points susceptibles d'être dénombrés, ils présentent un phénomène remarquable que je voudrais faire connaître dans ce qui va suivre. Considérons un système de points quelconque $(M)$ condensé dans toute l'étendue d'un ensemble continu $G_{n}$ à $n$ dimensions, 
et jouissant de la propriété de pouvoir être dénombré, en sorte qu'on peut représenter les points appartenant à $(M)$ sous forme de série:

$$
M_{1}, M_{2}, \ldots \ldots M_{\nu}, \ldots \ldots
$$

prenons comme exemple, dans notre espace à trois dimensions, le système de tous les points dont les coordonnées, par rapport à un système orthogonal de coordonnées $x, y, z$, sont toutes trois des nombres algébriques. Imaginons le système dénombrable de points $(M)$, enlevé de l'ensemble $G_{n}$ et désignons par $A$ l'ensemble qui reste alors; nous avons ce théorème remarquable, que: pour $n \overline{2}$ l'ensemble $A$ ne cesse pas d'être continu et connexe; en d'autres termes, que: deux points quelconques $N$ et $N^{\prime}$ de l'ensemble $A$ peuvent toujours être réunis par une ligne continue qui appartient, avec tous ses points, à l'ensemble $A$, en sorte qu'elle ne contient pas un seul point du système $(M)$.

Il suffit de reconnaitre la vérité de ce théorème pour le cas $n=2$; sa démonstration repose essentiellement sur le théorème démontré dans l'art. I, que: si on a une série régulière quelconque de grandeurs réelles:

$$
u_{1}, u_{2}, \ldots \ldots u_{\nu}, \ldots \ldots,
$$

(parmi lesquelles il peut y en avoir qui soient égales, ce qui évidemment ne change rien au théorème), on peut trouver dans chaque intervalle $(\alpha \ldots \ldots \beta)$ donné arbitrairement, et si petit qu'on le suppose, des grandeurs réelles $v$, qui ne se présentent pas dans cette série.

Soit en effet $G_{2}^{\prime}$ une portion continue quelconque du plan indéfini; prenons dans $G^{\prime}{ }_{2}$ le système de points $(M)$ supposé dénombrable et condensé dans toute l'étendue de $G^{\prime}{ }_{2}$; soient enfin $N$ et $N^{\prime}$ deux points quelconques de la portion continue $G_{2}^{\prime}$, n'appartenant pas au système $(M)$ et que nous relions d'abord l'un ì l'autre par une ligne continue $l$ comprise dans l'intérieur de $G_{2}^{\prime}$, sans nous inquiéter des points $(M)$; il faut montrer maintenant que la ligne $l$ peut être remplacée par une autre ligne continue $l^{\prime}$, qui relie aussi l'un à l'autre les points $N$ et $N^{\prime}$, qui est aussi comprise dans les limites de $G_{2}^{\prime}$, mais qui ne contient pas un seul point du système $(M)$.

En général il y aura sur $l$ un nombre infini de points da système $(\boldsymbol{M})$, en tout cas ils forment sur cette ligne une partie de $(\boldsymbol{M})$, par conséquent aussi un système susceptible d'être dénombré. 
Par suite du théorème d'arithmétique qui vient d'être mentionné, il $y$ a donc dans chaque intervalle de la ligne $l$, si petit qu'il soit, des points qui n'appartiennent pas à $(M)$. Considérons un nombre fini $N_{1}$, $N_{2}, \ldots \ldots N_{x}$, de ces points de la ligne $l$, tels que les segments de droites $N N_{1}, N_{1} N_{2}, \ldots \ldots N_{x} N^{\prime}$ soient aussi comprises en entier dans l'intérieur de $G_{2}^{\prime}$ On peut toujours. remplacer ces segments par des ares de cercle ayant les mêmes points extrèmes, compris aussi dans les limites de $G_{2}^{\prime}$, ne renfermant pas un seul point du système $(M)$ et formant, par leur réunion, une ligne continue $l^{\prime}$ ayant les caractères décrits plus haut.

Il suffira de démontrer cette proposition pour un des segments, par exemple pour le premier $N N_{1}$.

Les cercles qui passent par les points $N$ et $N_{1}$ forment un groupe continu, simplement infini; leurs centres sont sur une droite déterminée $g$; déterminons la position d'un de ces centres par sa distance $u$ à un point fixe $O$ de la droite $g$, cette distance étant affectée d'un signe; on peut alors en tout cas faire varier $u$ dans un intervalle $(\alpha \ldots \ldots \beta)$ tel que, pour chaque cercle correspondant à un de ces $u$, un des deux arcs de cercle qui relient $N$ et $N_{1}$ se trouve tout entier dans l'ensemble $G_{2}^{\prime}$.

Les centres des cercles de notre groupe qui passent par les points:

$$
M_{1}, M_{2}, \ldots \ldots M_{\nu}, \ldots \ldots
$$

du système $\boldsymbol{M}$, forment sur la droite $g$ un système de points susceptible d'être dénombré:

$$
P_{1}, P_{2}, \ldots P_{\nu}, \ldots \ldots
$$

les valeurs correspondantes de $u$ étant:

$$
u_{1}, u_{2}, \ldots \ldots u_{\nu}, \ldots \ldots
$$

$\mathrm{Si}$ on prend alors dans l'intervalle $(\alpha \ldots \ldots \beta)$ un nombre $v$ qui ne soit égal à aucun $u_{\text {e }}$ (ce qu'on peut toujours faire d'après le théorème cité), on obtient, en faisant $u=v$, un cercle du groupe, sur la circonférence duquel ne se trouve pas un seul point du système $(M)$ et qui, à cause de $\alpha<v<\beta$, nous présente un arc de cercle reliant les points $N$ et $N_{1}$, dans les conditions demandées.

Il est donc démontré que, étant donnés deux points quelconques $N$ et $N^{\prime}$ de l'ensemble $A^{\prime}$, qui reste de l'ensemble $G_{2}^{\prime}$ après qu'on en a 
enlevé un système de points $(M)$ condensé dans toute son étendue et susceptible d'être dénombré, on peut relier ces deux points par une courbe continue $l^{\prime}$ composée d'un nombre fini d'arcs de cercle, et appartenant avec tous ses points à l'ensemble $A^{\prime}$, c. à d. ne contenant pas un seul point du système $(M)$.

Du reste on pourrait aussi, par le même moyen; relier les points $N$ et $N^{\prime}$ par une ligne continue se développant d'après une loi analytique unique, et comprise tout entière dans l'ensemble $A^{\prime}$.

A ces théorèmes se rattachent des considérations sur la nature de l'espace réel à trois dimensions qui doit servir de base à la description et à l'explication des phénomènes qui se présentent dans le monde réel. On sait que cet espace, soit à cause des formes qui s'y rencontrent, soit surtout à cause des mouvements qui y ont lieu, est considéré comme généralement continu. D'après les travaux publiés en même temps, mais indépendants l'un de l'autre, de Dederind (V. l'opuscule: La continuité et les nombres irrationnels, R. Dederind, Brunswick, 1872) et de l'auteur, cette dernière supposition consiste seulement en ce que tout point, dont les coordonnées $x, y, z$ par rapport à un système de coordonnées rectangulaire sont fournies par des nombres réels déterminés quelconques, rationnels ou irrationnels, est considéré comme appartenant réellement à l'espace; il n'y a à cela aucune nécessité intrinsèque et il n'y faut voir qu'une construction arbitraire, quoique légitime. L'hypothèse de la continuité de l'espace n'est donc rien autre chose que la supposition, arbitraire en elle-même, de la correspondance complète, réciproque et à sens unique entre le continu purement arithmétique à trois dimensions $(x, y, z)$ et l'espace qui sert de base au moñde des phénomènes.

Nous pouvons facilement par la pensée faire abstraction de points isolés de l'espace, même quand ils sont condensés dans toute une étendue, et aboutir à la notion d'un espace discontinu à trois dimensions $A$, dans les conditions décrites plus haut. Quant à la question qui se présente alors, de savoir si on peut aussi imaginer un mouvement continu dans des espaces ainsi discontinus, il faut, d'après ce qui précède, y répondre affirmativement, et d'une manière absolue; car nous avons montré qu'on peut relier deux points quelconques d'une figure $A$ par un nombre infini de lignes continues parfaitement regulières. On arrive donc à cette conséquence remarquable qu'on ne peut rien conclure immédiatement, du seul 
fait du mouvement continu, pour la continuité générale de l'espace à trois dimensions, tel qu'on l'a conçu pour expliquer les phénomènes du mouvement. On peut donc entreprendre l'essai d'une mécanique modifiée, applicable aux espaces de la même nature que $A$ : grâce aux résultats de ces recherches, que l'on comparera avec les faits, on arrivera peut-ètre à obtenir des points d'appui réels pour l'hypothèse de la continuite générale de l'espace, tel qu'on le conçoit dans la pratique.

Berlin, le 31 Mars 1882. 


\title{
SUR LES ENSEMBLES INFINIS ET LINÉAIRES \\ DE POINTS
}

PAR

\author{
G. CANTOR
}

à HALIE a. s.

\section{IV.}

(Traduction d'un mémoire publié dans les Annales mathématiques de Leipsic, t. XXI, p. 51.)

Nous avons maintenant à énoncer et à démontrer divers théorèmes nouveaux qui se rattachent aux développements donnés précédemment, et qui sont à la fois intéressants en eux-mêmes et utiles pour la théorie des fonctions. Nous nous servirons de la notation suivante.

Soient plusieurs ensembles de points $P_{1}, P_{2}, P_{3}, \ldots$ qui n'ont deux à deux aucun point commun, et $P$ le système résultant de leur réunion, nous choisirons, au lieu des formules employées plus haut, (t. XVII, p. 355), la formule plus commode:

$$
P \equiv P_{1}+P_{2}+P_{3}+\ldots \ldots
$$

Et par conséquent, $Q$ étant un ensemble contenu dans $P$ et $R$ le système qui reste quand on enlève $Q$ de $P$, on pourrait écrire:

$$
R \equiv P-Q .
$$

Un système de points $Q$, que nous nous représentons dans un espace continu à $n$ dimensions, peut ètre dans des conditions telles qu'aucun des. 
points qui lui appartiennent ne soit en même temps un point-limite; nous donnerons à ce système, pour lequel

$$
\mathfrak{D}\left(Q, Q^{\prime}\right) \equiv 0,
$$

le nom de système de points isolé. Si l'on a un système de points quelconque non isolé $P$, on peut en tirer un système isolé $Q$, en enlevant de $P$ le système $\mathfrak{D}\left(P, P^{\prime}\right)$.

On. a donc:

$$
Q \equiv P-\mathscr{D}\left(P, P^{\prime}\right)
$$

et par conséquent:

$$
P \equiv Q+\mathfrak{D}\left(P, P^{\prime}\right)
$$

Tout ensemble de points peut donc être composé d'un ensemble isolé $Q$ et d'un autre ensemble $R$, qui est diviseur de l'ensemble dérivé $P^{\prime}$. Si nous remarquons ensuite, ce qui a déjà été signalé souvent, que chaque dérivé supérieur d'un système $P$ est contenu dans le dérivé précédent, nous voyons que:

$$
P^{\prime}-P^{\prime \prime}, P^{\prime \prime}-P^{\prime \prime \prime}, \ldots \ldots P^{(\nu)}-P^{(\nu+1)}, \ldots
$$

sont tous des systèmes isolés.

Mais on a les décompositions, très-importantes pour ce qui doit suivre:

$$
P^{\prime} \equiv\left(P^{\prime}-P^{\prime \prime}\right)+\left(P^{\prime \prime}-P^{\prime \prime \prime}\right)+\ldots \ldots+\left(P^{(n-1)}-P^{(n)}\right)+P^{(n)}
$$

et

$$
P^{\prime} \equiv\left(P^{\prime}-P^{\prime \prime}\right)+\left(P^{\prime \prime}-P^{\prime \prime \prime}\right)+\ldots .+\left(P^{(\nu-1)}-P^{(\nu)}\right)+\text { à l'infini }+P^{(\omega)} \text {. }
$$

Maintenant le théorème suivant est vrai pour les systèmes de points isolés:

Théorème $\boldsymbol{I}$. Tout ensemble de points isolé peut être dénombré, et appartient par conséquent à la première classe.

Démonstration. Soient $Q$ un système de points isolé quelconque compris dans un espace $\grave{a} n$ dimensions, $q$ un point de ce système, $q^{\prime}$, $q^{\prime \prime}, q^{\prime \prime \prime} \ldots$ les autres points de $Q$.

Les distances $\overline{q q^{\prime}}, \overline{q q^{\prime \prime}}, \overline{q q^{\prime \prime \prime}}, \ldots \ldots$ ont une limite inférieure, que je désigne par $\rho$. 
Soient de inềme $\rho^{\prime}$ la limite inférieure des distances $\overline{q^{\prime} q}, \overline{q^{\prime} q^{\prime \prime}}, \overline{q^{\prime} q^{\prime \prime \prime}}, \ldots ., \rho^{\prime \prime}$ la limite inférieure des distances $\overline{q^{\prime \prime} q}, \overline{q^{\prime \prime} q^{\prime}}, \overline{q^{\prime \prime} q^{\prime \prime \prime}}, \ldots$. . etc.

Toutes ces grandeurs $\rho, \rho^{\prime}, \rho^{\prime \prime}, \rho^{\prime \prime \prime} \ldots$ sont distinctes de zéro, parce que $Q$ est un ensemble isolé.

Qu'on trace, avec $q$ comme centre, la figure à $(n-1)$ dimensions, dont les points sont à la distance $\frac{\rho}{2}$ de $q$; cette figure borne une sphère pleine à $n$ dimensions, que nous désignerons par $K$. Qu'on forme de mếme une sphère pleine $K^{\prime}$ ayant pour centre le point $q^{\prime}$ et poúr rayon $\frac{\rho^{\prime}}{2}$, une sphère pleine $K^{\prime \prime}$ ayant pour centre le point $q^{\prime \prime}$ et pour rayon $\frac{\rho^{\prime \prime}}{2}$, etc.

Il est maintenant essentiel de remarquer que deux quelconques de ces sphères pleines, par ex. $K$ et $K^{\prime}$ peuvent tout au plus être tangentes entre elles, mais sont d'ailleurs complètement extérieures l'une à l'autre.

Cela dérive de ce que, d'après la définition des grandeurs $\rho$ et $\rho^{\prime}$, elles sont plus petites que $\overline{q q^{\prime}}$ ou égales à $\overline{q q^{\prime}}$, et que par conséquent les rayons $\frac{\rho}{2}, \frac{\rho^{\prime}}{2}$ des deux sphères $K$ et $K^{\prime}$ ne sont pas plus grands que la moitié de la ligne des centrés $\overline{q q^{\prime}}$.

Par conséquent les sphères pleines $K, K^{\prime}, \ldots$ forment un ensemble de portions, extérieures l'une à l'autre et à $n$ dimensions, de l'espace à $n$ dimensions que nous avons pris pour base; mais un ensemble de cette espèce peut toujours être dénombré, comme on l'a démontré t. XX, p. 117 . Par conséquent les centres $q, q^{\prime}, q^{\prime \prime}, \ldots$ forment aussi un système susceptible d'être dénombré, c. à d. que $Q$ peut être dénombré.

Nous pouvons maintenant énoncer les théorèmes suivants.

Théorème II. Si le dérivé $P^{\prime}$ d'un ensemble de points $P$ peut être dénombré, $P$ jouit aussi de la même propriété.

Démonstration. Qu'on désigne par $R$ le plus grand commun diviseur de $P$ et de $P^{\prime}$, en sorte que:

$$
R \equiv \mathfrak{D}\left(P, P^{\prime}\right)
$$

et qu'on pose:

$$
P-R \equiv Q
$$

$Q$ est alors, comme nous l'avons vu plus haut, un ensemble isolé, et par conséquent susceptible d'ẹtre dénombré d'après le théorème I. 
Sur les ensembles infinis et linéaires de points.

$R$ jouit de la même propriété, comme élément constitutif du système $P^{\prime}$, susceptible d'être dénombré, d'après l'hypothèse.

La réunion de deux systèmes susceptibles d'être dénombrés donne toujours lieu à 'un nouveau système qu'on peut également dénombrer; par conséquent $P \equiv Q+R$ est susceptible d'ệtre dénombré.

Théorème III. Tout ensemble du premier genre et de la $n^{\text {ème }}$ espèce peut être dénombré.

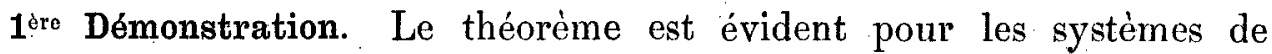
points d'espèce 0 qui sont évidemment des systèmes de points isolés. Mais nous allons développer complètement l'induction, en supposant le théorème vrai pour les systèmes de points de $0^{\text {ème }}$, de $1^{\text {ère }}$, de $2^{\text {ème }}, \ldots$. de $(n-1)^{\text {ème }}$ espèce, et nous allons montrer, avec cette hypothèse, qu'il est vrai aussi pour les systèmes de points de la $n^{\text {ème }}$ espèce.

Soit $P$ un système de points de la $n^{\text {ème }}$ espèce, $P^{\prime}$ sera de la $(n-1)^{\text {ème }}$ espèce; $P^{\prime}$ est donc susceptible d'être dénombré, d'après l'hypothèse, et par conséquent $P$ l'est aussi d'après le théorème II.

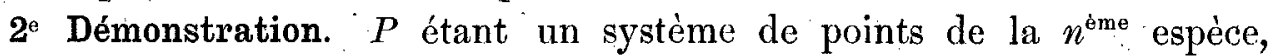
$P^{(n)}$ sera de l'espèce 0 , et par conséquent un système de points isolé.

On a alors:

$$
P^{\prime} \equiv\left(P^{\prime}-P^{\prime \prime}\right)+\left(P^{\prime \prime}-P^{\prime \prime \prime}\right)+\ldots .+\left(P^{(n-1)}-P^{(n)}\right)+P^{(n)} .
$$

Tous les éléments du côté droit $\left(P^{\prime}-P^{\prime \prime}\right)\left(P^{\prime \prime}-P^{\prime \prime \prime}\right), \ldots\left(P^{(n-1)}-P^{(n)}\right)$ et $P^{(n)}$ sont des ensembles isolés, par conséquent tous susceptibles d'ètre dénombré d'après le théorème I; le système $p^{\prime}$ formé par leur réunion, est donc susceptible d'étre dénombré et, d'après le théorème II, $P$ le sera aussi.

Théorème IV. Tout système de points du deuxième genre, pour lequel $P^{(\omega)}$ est susceptible d'ètre dénombré, jouit aussi de la même propriété:

La démonstration de ce théorème ressort de la décomposition suivante:

$$
P^{\prime} \equiv\left(P^{\prime}-P^{\prime \prime}\right)+\ldots .+\left(P^{(\nu-1)}-P^{(\omega)}\right)+\ldots \text { à l'infini }+P^{(\omega)}
$$

En effet comme tous les éléments de droite sont susceptibles d'être dénombrés et que l'ensemble de ces éléments est de la première puissance, on tire de là pour $P^{\prime}$ et d'après le théorème II pour $P$, la propriété de pouvoir être dénombré. 
$\mathrm{Si}$ on désigne par $\alpha$ in quelconque des symboles d'infini introduits t. XVII p. 357 , on a le théorème plus général:

Théorème $\boldsymbol{V}$. Tout systèmè de points $P$ du deuxième genre pour lequel $P^{(\alpha)}$ est susceptible d'être dénombré, jouit aussi de la même propriété.

Ce théorème se démontre, à l'aide de l'induction complète, comme les théorèmes III et IV.

On peut aussi formuler les derniers théorèmes de la manière suivante: $P$ étant un" système de points non-susceptible d'être dénombré, $P^{(\alpha)}$ ne le sera pas non plus, soit que $\alpha$ soit un nombre entier fini, ou un des symboles d'infini.

Dans leurs travaux sur certaines généralisations de théorèmes du calcul intégral, M.M. Du Bors-Reymond et Harnack emploient des systèmes de points linéaires que l'on peut renfermer dans un nombre fini d'intervalles, en sorte que la somme de tous les intervalles est plus petite qu'une grandeur donnée à volonté.

Pour qu'un système de points linéaire jouisse de cette propriété, il faut évidemment qu'il ne soit condensé dans toute l'étendue d'aucun intervalle, si petit qu'il soit; cependant cette dernière condition ne parait pas suffisante pour qu'un systeme de points soit tel que nous venons de le dire. En revanche nous pouvons démontrer le théorème suivant.

Théorème VI. Un système de points linéaire $P$ contenu dans un intervalle $(a, b)$ étant constitué de telle sorte que son ensemble dérivé $P^{\prime}$ soit susceptible d'être dénombré, on peut toujours renfermer $P$ dans un nombre fini d'intervalles, la somme de ces intervalles étant aussi petite que l'on voudra.

Dans la démonstration qui va suivre nous nous servirons des théorèmes auxiliaires ci-dessous, dont le premier exprime une propriété connue des fonctions continues, et les deux autres sont le résultat de nos considérations précédentes.

Théorème auxiliaive $I$. Une fonction continue $\varphi(x)$ donnée dans un intervalle $(c, d)$ de la variable continue $x$, et ayant à ses limites des valeurs inégales $\varphi(c)$ et $\varphi(d)$, prend une fois au moins une valeur $y$ comprise entre les limites $\varphi(c)$ et $\varphi(d)$.

Théorème auxiliaive II. Un nombre infini d'intervalles, dans une droite infinie, extérieurs l'un à l'autre, et ne se rencontrant tout au plus qu’à leurs limites, est toujours susceptible d'ètre dénombré. 
Théorème anxiliaive III. Si l'on a un ensemble de grandeurs, qui est de la première puissance:

$$
u_{1}, u_{2}, \ldots \ldots u_{\nu}, \ldots \ldots
$$

on peut, dans tout intervalle proposé, trouver une grandeur $v$ qui ne se rencontre pas parmi ces grandeurs.

Démonstration du théorème VI. Prenons, pour simplifier, l'intervalle $(a, b)$, qui comprend $P$, de sorte que $a=0, b=1$; on peut facilement, par une transformation, ramener le cas général à ce cas particulier. $P$ se trouve donc dans l'intervalle $(0,1)$; la même chose est évidemment. vraie pour $P^{\prime}$ et pour le système produit par la réunion des points de $P$ et $P^{\prime}$ et que nous désignerons par $Q$.

On a:

$$
Q \equiv \mathfrak{M}\left(P, P^{\prime}\right)
$$

Nous désignons ensuite par $R$ le système de points compris dans l'intervalle $(0,1)$ et qui est constitué par les points restants dans cet intervalle après qu'on en a enlevé le système $Q$, en sorte que:

$$
(0,1) \equiv Q+R \text {. }
$$

De ce que le système $P^{\prime}$ est susceptible d'être dénombré, comme on l'a supposé, on tire d'abord les conclusions suivantes:

1. $P$ est aussi susceptible d'être dénombré, d'après le théorème II, par conséquent il en est de même de $Q$.

2. $P$ et par conséquent $P^{\prime}$ ne sont condensés dans toute l'étendue d'aucun intervalle; car si $P$ était condensé dans toute l'étendue de l'intervalle $(i, k)$, tous les points de cet intervalle appartiendraient à $P^{\prime}$ et, d'après le théorème auxiliaire III, $P^{\prime}$ ne pourrait pas être dénombré. Par conséquent $Q$ n'est condensé dans toute l'étendue d'aucuin intervalle. Les valeurs des coordonnées, qui correspondent aux points du système $Q$, susceptible d'être dénombré, peuvent être appelées

$$
u_{1}, u_{2}, \ldots \ldots u_{\nu}, \ldots \ldots
$$

$\mathrm{Si}$ maintenant nous considérons le système $R$, on peut montrer que les valeurs des coordonnées correspondant à ses points sont situées respectivement dans l'intérieur d'une série infinie d'intervalles: 


$$
\left(c_{1}, d_{1}\right),\left(c_{2}, d_{2}\right), \ldots \ldots\left(c_{\nu}, d_{\nu}\right), \ldots \ldots
$$

extérieurs l'un à l'autre et compris dans l'intervalle $(0,1)$. Comme les valeurs intérieures à ces intervalles appartiennent seules à des points du système $R$, il résulte de la relation (1) que les limites $c_{\nu}$ et $d_{y}$ de ces intervalles correspondent à des points du système $Q$, et par conséquent se présentent dans la série (2).

En effet, soit $r$ un point de $R$, les points de $Q$ ne peuvent pas se rapprocher à l'infini de $r$, parce qu'autrement $r$ serait point-limite de $P$ et par conséquent appartiendrait à $Q$. Il doit maintenant y avoir à gauche de $r$ un point $c$ et à droite de $r$ un point $d$, tels qu'aucun point de $Q$ ne se trouve dans l'intervalle $(c, d)$ et que par contre il y ait en dehors de cet intervalle, des points de $Q$ aussi rapprochés qu'on le voudra de $c$ et de $d$, au cas où $c$ et $d$ ne sont pas des points isolés de $Q$; mais comme chaque point-limite de $Q$ appartient à $Q, c$ et $d$, même dans le dernier cas, appartiennent aussi $\stackrel{a}{Q}$. Les intervalles en nombre infini $(c, d)$, ainsi obtenus, sont tous, évidemment, extérieurs l'un à l'autre et forment par conséquent, d'après le théorème auxiliaire II, un système susceptible d'ètre dénombré (3), ce qu'il fallait démontrer.

Puisque nous supposons $c_{\nu}<d_{\nu}$, la grandeur de l'intervalle $\left(c_{\nu}, d_{\nu}\right)$ est:

$$
=d_{\nu}-c_{\nu} \text {. }
$$

La somme, de toutes ces grandeurs d'intervalles s'appellera $\sigma$, en sorte que:

$$
\sum_{\nu=1}^{\infty}\left(d_{\nu}-c_{\nu}\right)=\sigma
$$

- On voit a priori que $\sigma \bar{\gtrless} 1$, parce que les intervalles sont tous extérieurs l'un à l'autre et sont contenus dans l'intervalle $(0,1)$. Si nous pouvions prouver que $\sigma=1$, notre théorème VI serait démontré, comme on peut s'en convaincre par une considération très-simple se rattachant au sens des intervalles $\left(c_{\nu}, d_{\nu}\right)$.

Toute notre démonstration se réduit donc à prouver que l'hypothèse $\sigma<1$ conduit à une contradiction.

Pour cela nous définissons, pour $0<x \bar{\gtrless} 1$, une fonction $f(x)$ comme il suit: Qu'on additionne les grandeurs de tous les intervalles $\left(c_{y}, d_{\nu}\right)$, 
tant que ces intervalles tombent dans les limites de l'intervalle $(0, x)$ et qu'on pose cette somme $=f(x)$. (On convient de ne prendre dans cette somme, d'un intervalle $\left(c_{\nu}, d_{\nu}\right)$ qui se trouve, en partie, en dehors de $(0, x)$, que la partie correspondante qui tombe dans les limites de $(0, x)$.) On a évidemment:

$$
f(1)=\sigma .
$$

Si de plus on établit que $f(0)=0$, il s'ensuit facilement que $f(x)$ est une fonction continue de $x$ pour $0 \leqq x \leqq 1$.

En effet de la définition de $f(x)$ il résulte immédiatement que, $x$ et $x+h$ étant deux valeurs distinctes de l'intervalle $(0,1)$, on a pour des valeurs positives de $h$ :

$$
f(x+h)-f(x) \stackrel{>}{\leqq}
$$

De là on conclut la continuité de $f(x)$.

On voit alors aussitót, en revenant à la définition de $f(x)$, que, si $x$ et $\dot{x}+h$ sont deux valeurs distinctes d'un seul et même intervalle partiel $\left(c_{\nu} ; d_{\nu}\right)$, on a:

$$
f(x+h)-f(x)=h,
$$

par conséquent aussi:

$$
(x+h)-f(x+h)=x-f(x) .
$$

- Si donc on introduit la fonction

$$
\varphi(x)=x-f(x),
$$

$\varphi(x)$ sera aussi une fonction continue de $x$ qui change sans diminuer de 0 à $1-\sigma$, si $x$ croìt de 0 à 1 . Ce changement se fait de telle façon que, dans les limites d'un des intervalles partiels $\left(c_{\nu}, d_{\nu}\right)$, la fonction continue $\varphi(x)$ conserve une valeur constante.

De là résulte pour la fonction $\varphi(x)$ cette propriété que: toutes les valeurs qu'elle prend sont épuisées par la série de valeurs:

$$
\varphi\left(u_{1}\right), \varphi\left(u_{2}\right), \ldots \varphi \varphi\left(u_{\nu}\right), \ldots \ldots
$$

En effet $x$ peut être égalé à une des valeurs $u$, et dans ce cas nous avons: 


$$
\varphi(x)=\varphi\left(u_{\nu}\right) \text {. }
$$

Ou bien $x$ est une valeur comprise dans un des intervalles $\left(c_{\nu}, d_{\nu}\right)$; dans ce cas, à cause de la constance de $\varphi(x)$ dans un de ces intervalles, nous avons:

$$
\varphi(x)=\varphi\left(c_{\nu}\right)=\varphi\left(d_{\nu}\right)
$$

Mais maintenant, comme nous l'avons vu plus haut, les valeurs $c_{\nu}$ et $d_{\nu}$ appartiennent également à. la série (2), on a par exemple:

$$
c_{\nu}=u_{\lambda} \text {. }
$$

Par conséquent on a aussi dans ce cas:

$$
\varphi(x)=\varphi\left(u_{\lambda}\right) \text {. }
$$

La - série (5) comprend donc toutes les valeurs que peut prendre généralement $\varphi(x)$.

Le système de valeurs, que peut prendre la fonction continue $\varphi(x)$, est par conséquent susceptible d'être dénombré.

Si maintenant $\sigma<1$, et par suite $1-\sigma$ distinct de zéro, la fonction continue $\varphi(x)$, d'après le théorème auxiliaire I prendrait au moins une fois toute valeur $y$ entre 0 et $1-\sigma$. Par conséquent, dans la série (5) qui épuise toutes les valeurs prises par la fonction $\varphi(x)$, comme on vient de le montrer, on trouverait tous les nombres possibles de l'intervalle $(0,1-\sigma)$, ce qui est en contradiction avec le théorème auxiliaire III. Il ne reste donc que l'hypothèse $\sigma=1$, ce qu'il fallait démontrer.

Hartzbourg, $1^{\text {er }}$ Septembre 1882. 